International Journal of Pure and Applied Mathematics

Volume 96 No. 1 2014, 79-104

ISSN: 1311-8080 (printed version); ISSN: 1314-3395 (on-line version)

url: http://www.ijpam.eu

doi: http://dx.doi.org/10.12732/ijpam.v96i1.7

ijpam.eu

\title{
MELLIN TRANSFORM METHOD FOR THE VALUATION \\ OF SOME VANILLA POWER OPTIONS WITH NON-DIVIDEND YIELD
}

\author{
Chuma Raphael Nwozo ${ }^{1}$, Sunday Emmanuel Fadugba ${ }^{2}$ \\ ${ }^{1}$ Department of Mathematics \\ University of Ibadan \\ Oyo State, NIGERIA \\ ${ }^{2}$ Department of Mathematical Sciences \\ Ekiti State University \\ Ado Ekiti, NIGERIA
}

\begin{abstract}
This paper presents the Mellin transform method for the valuation of some vanilla power options with non-dividend yield. This method is a powerful tool used in the valuation of options. We extend the Mellin transform method proposed by Panini R. and Srivastav R.P. [15] to derive the price of European and American power put options with non-dividend yield. We also derive the fundamental valuation formula known as the Black-Scholes model using the convolution property of the Mellin transform method. To provide a sufficient numerical analysis, we compare the results generated by the Mellin transform method for the valuation of American power put option for $n=1$ which pays no dividend yield to two other numerical methods namely Crank Nicolson finite difference method [2] and binomial model [3] for options valuation against Black-Scholes analytical pricing formula [1]. The numerical experiment shows that the Mellin transform method is efficient, easy to implement, agree with the values of Black-Scholes [1], Crank Nicolson finite difference method [2] and binomial model [3]. Hence the Mellin transform method is a better alternative method compared to the Crank Nicolsion finite difference and binomial model for the valuation of some vanilla power options.
\end{abstract}

Received: June 2, 2014

(C) 2014 Academic Publications, Ltd.

${ }^{\S}$ Correspondence author url: www.acadpubl.eu 
AMS Subject Classification: 44A15, 44A99, 60H30, 91G20, 91G99

Key Words: American option, European power option, Mellin transform method, non-dividend yield

\section{Introduction}

The derivative market has becomes extremely popular, this popularity even exceeds that of the stock exchange. Many problems in mathematical finance entail the computation of a particular integral. In many cases these integrals can be valued analytically and in some cases they can be valued using numerical integration, or computed using a partial differential equation. Option price estimation as the most interesting of the derivatives has many approaches and diverse qualities.

An option is an instrument whose value derives from that of another asset; hence it is called a "derivative". In other words an option on an underlying asset is an asymmetric contract that is negotiated today with the following conditions in the future. The holder has either the right, but not the obligation to buy, as it is the case with the European call option, or the possibility to sell, as in the case of the European put option, an asset for a certain price at a prescribed date in the future. The American type of option can be exercised at any time up to and including the date of expiry. The distinctive features of American option is its early exercise privilege. The pricing of American options has been the subject of extensive research in the last decades. There is no known closed form solution and many numerical and analytic approximations have been proposed. Black F. and Scholes M. [1] published their seminal work on option pricing in which they described a mathematical frame work for finding the fair price of a European option. They used a no-arbitrage argument to describe a partial differential equation which governs the evolution of the option price with respect to the maturity time and the price of the underlying asset. In the recent years the complexity of numerical computational in financial theory and practice has increased greatly, putting more demands on computation speed and efficiency. The subject of numerical methods in the area of options valuation and hedging is very broad. A wide range of different types of contracts are available and in many cases there are several candidate models for the stochastic evolution of the underlying state variables.

We present an overview of Mellin transform method for the valuation of options in the context of Black F. and Scholes M. [1]. The Mellin transform is an integral transform named after the Finnish mathematician Hjalmar Mellin 
(1854-1933). Mellin transforms in option theory were introduced by Panini R. and Srivastav R.P. [14]. They derived the expression for the free boundary and price of an American perpetual put as the limit of a finite-lived option. In this contribution Frontczak F. and Schöbel [7] extended the results obtained in Panini R. and Srivastav R.P. [14] and showed how the Mellin transform approach can be used to derive the valuation formula for perpetual American put options on dividend-paying stocks. Panini R. and Srivastav R.P. [15] derived integral equation representations for the price of European and American basket put options using Mellin transform techniques. Samuelson P.E. [16] derived a closed-form expression for the free boundary and price of a perpetual American put option using Mellin transform techniques, Frontczak F. and Schöbel [8] extended a framework based on the Mellin transforms and showed how to modify the approach to value American call options on dividend paying stocks, Zieneb A.E and Rokiah R.A [21] derived a closed form solution for a continuous arithmetic Asian option by means of partial differential equation. They also provided a new method for solving arithmetic Asian options using Mellin transforms in a stock price. Vasilieva O. [17] introduced a new method of pricing Multi-options using Mellin transforms and integral equations.

For mathematical backgrounds, Mellin transform method in derivatives pricing and some numerical methods for options valuation see [2], [3], [4], [5], $[6],[8][9],[10],[11],[12],[13],[16],[17],[18],[19],[20]$ just to mention a few. In this paper, we focus on Mellin transform method for the valuation of some vanilla power options with non-dividend yield. This paper is structured as follows: Section 2 presents the power options and a review of classic integral transforms. Section 3 presents some operational fundamental properties of Mellin transform method, derivation of Black-Scholes Formula for the valuation of European power put option using Mellin transform method and Mellin transform method for the valuation of European and American power put options. Section 4 concludes the paper.

\section{Power Options}

Power option is defined as a contingent claim on the product of powers of several assets. It can also be seen as a class of options in which the payoff at expiry is related to the $n^{\text {th }}$ power of the underlying price of the asset, where $n \in\left\{Z^{+}-0\right\}$. American power option is an option that can be exercised before or at the expiry date with non-linear payoff. European power option is an option that can be exercised only at the expiry date with non-linear payoff. 
European power option comes in two forms namely European power call and put options. A European power call option is an option with non-linear payoff given by the difference between price of the underlying asset at maturity raised to a strictly positive power and the exercise price. A European power put option is an option with non-linear payoff given by the difference between the exercise price and price of the underlying asset at maturity raised to a strictly positive power. For a European power option on the underlying price of the asset $S_{t}$ with exercise price $K$ and time to expiry $T$, we have the payoff for the European power call option as

$$
E_{c}^{n}\left(S_{T}^{n}, T\right)=\max \left(S_{T}^{n}-K, 0\right)=\left(S_{T}^{n}-K\right)^{+}
$$

The payoff for the European power put option is given as

$$
E_{p}^{n}\left(S_{T}^{n}, T\right)=\max \left(K-S_{T}^{n}, 0\right)=\left(K-S_{T}^{n}\right)^{+}
$$

\section{Remark 2.0.1:}

- For $n>0$, European power option allows parties to negotiate the underlying price of the asset, exercise price, expiration date and other features. It also gives investors the opportunity to trade on a large scale with expanded or eliminated position limit and is of practical interest since OverThe-Counter (OTC) traded options exhibit such a payoff structure.

- The payoff curves for European power call and put options become concave and thus have a negative time value for $n<1$ and $n>1$ respectively, i.e. current price of the underlying asset $\left(S_{t}^{n}\right)<$ payoff $\left(E_{p}^{n}(S, T)\right)$.

- For $n=1$, the payoffs for European power call and put options in (1) and (2) become (3) and (4) respectively

$$
\begin{aligned}
& E_{c}^{1}(S, T)=E_{c}(S, T)=\max \left(S_{T}^{1}-K, 0\right)=\left(S_{T}^{1}-K\right)^{+} \\
& E_{p}^{1}(S, T)=E_{p}(S, T)=\max \left(K-S_{T}^{1}, 0\right)=\left(K-S_{T}^{1}\right)^{+}
\end{aligned}
$$

Equations (3) and (4) give the payoffs for regular European call and put options respectively and can also be expressed as

$$
E_{c}(S, T)=\max \left(S_{T}-K, 0\right)=\left(S_{T}-K\right)^{+}, \text {for the call option }
$$

and

$$
E_{p}(S, T)=\max \left(K-S_{T}, 0\right)=\left(K-S_{T}\right)^{+}, \text {for the put option }
$$

respectively. 
- As $n$ increases, the value of European power put option becomes very large.

Theorem 2.0.1: Let $S_{t}^{n}$ denote the price of the underlying asset, $\sigma$ the volatility, $r$ the risk interest rate, $n$ the power of the option and $W_{t}$ the Wiener process or Brownian motion. If the underlying price of the asset $S_{t}^{n}$ follows a random process in

$$
d S_{t}^{n}=\left(n r+\frac{1}{2} n(n-1) \sigma^{2}\right) S_{t}^{n} d t+n \sigma S_{t}^{n} d W_{t},
$$

then the explicit formula for the evolution of the underlying price of the asset is given by

$$
S_{T}^{n}=S_{0}^{n} \exp \left[n\left(r-\frac{1}{2} \sigma^{2}\right) T+n \sigma W_{T}\right]
$$

Proof: Let

$$
u=u\left(S_{t}^{n}, t\right)=\ln S_{t}^{n}
$$

Differentiating (7) we have

$$
\frac{\partial u}{\partial S_{t}^{n}}=\frac{1}{S_{t}^{n}}, \frac{\partial^{2} u}{\partial\left(S_{t}^{n}\right)^{2}}=\frac{-1}{\left(S_{t}^{n}\right)^{2}}, \frac{\partial u}{\partial t}=0
$$

Recall from Ito's lemma using (5) for any derivative $u\left(S_{t}^{n}, t\right)$ we have

$$
d u\left(S_{t}^{n}, t\right)=\left(\frac{\partial u}{\partial t}+f \frac{\partial u}{\partial S_{t}^{n}}+\frac{g^{2}}{2} \frac{\partial^{2} u}{\partial\left(S_{t}^{n}\right)^{2}}\right) d t+g \frac{\partial u}{\partial S_{t}^{n}} d W_{t}
$$

From (5), we can write for

$$
f=\left(n r+\frac{1}{2} n(n-1) \sigma^{2}\right) S_{t}^{n}, g=n \sigma S_{t}^{n}
$$

substituting (7), (8) and (10) into (9) and rearranging the terms, we have

$$
\begin{gathered}
d\left(\ln S_{t}^{n}\right)=\left(\left(n r+\frac{1}{2} n(n-1) \sigma^{2}\right) S_{t}^{n}\left(\frac{1}{S_{t}^{n}}\right)\right) d t+ \\
\left(\frac{1}{2} n^{2} \sigma^{2}\left(S_{t}^{n}\right)^{2}\left(\frac{-1}{\left(S_{t}^{n}\right)^{2}}\right)\right) d t+n \sigma S_{t}^{n}\left(\frac{1}{S_{t}^{n}}\right) d W_{t}
\end{gathered}
$$

Therefore,

$$
d\left(\ln S_{t}^{n}\right)=\left(n r-\frac{1}{2} n \sigma^{2}\right) d t+n \sigma d W_{t}
$$


Thus, $\ln S_{t}^{n}$ is a Brownian motion with drift parameter $\left(n r-\frac{1}{2} n \sigma^{2}\right)$ and variance parameter $n \sigma^{2}$. To derive an explicit formula for the evolution of the stock price, we integrate (12) from 0 to $T$ to obtain

$$
\begin{gathered}
\ln \left(\frac{S_{T}^{n}}{S_{0}^{n}}\right)=n\left(r-\frac{1}{2} \sigma^{2}\right) T+n \sigma W_{T} \\
S_{T}^{n}=S_{0}^{n} \exp \left[n\left(r-\frac{1}{2} \sigma^{2}\right) T+n \sigma W_{T}\right]
\end{gathered}
$$

(14) can also be written as

$$
S_{T}^{n}=S_{0}^{n} \exp \left[n\left(r-\frac{1}{2} \sigma^{2}\right) T+n \sigma Z \sqrt{T}\right]
$$

where $Z \sim N(0,1)$. Therefore the stock dynamic follows a log-normal distribution. For $n=1$, (15) becomes

$$
S_{T}^{1}=S_{0}^{1} \exp \left[\left(r-\frac{1}{2} \sigma^{2}\right) T+\sigma Z \sqrt{T}\right]
$$

(16) shows that plain vanilla option follows a log-normal distribution. The partial differential equation for any derivative $f$ written on $S_{t}^{n}$ is given by

$$
\frac{\partial f}{\partial t}+n\left(\frac{1}{2} \sigma^{2}(n-1)+r\right) S_{t}^{n} \frac{\partial f}{\partial S_{t}^{n}}+\frac{1}{2}\left(\sigma n S_{t}^{n}\right)^{2} \frac{\partial^{2} f}{\partial\left(S_{t}^{n}\right)^{2}}=r f
$$

\section{Remark 2.0.2:}

- This result shows that the price of the underlying asset follows a random process. Also an explicit formula for the evolution of the underlying price of the asset can be obtained.

- For $n=1,(17)$ is known as the regular Black-Scholes partial differential equation which is given by

$$
\frac{\partial f}{\partial t}+r S_{t} \frac{\partial f}{\partial S_{t}}+\frac{1}{2} \sigma^{2} S_{t}^{2} \frac{\partial^{2} f}{\partial S_{t}^{2}}=r f
$$

The European power put option with non-dividend yield can be obtained by setting $f=E_{p}^{n}\left(S_{t}^{n}, T\right)=E_{p}^{n}$ in (17) as

$$
\frac{\partial E_{p}^{n}}{\partial t}+n\left(\frac{1}{2} \sigma^{2}(n-1)+r\right) S_{t}^{n} \frac{\partial E_{p}^{n}}{\partial S_{t}^{n}}+\frac{1}{2}\left(\sigma n S_{t}^{n}\right)^{2} \frac{\partial^{2} E_{p}^{n}}{\partial\left(S_{t}^{n}\right)^{2}}-r E_{p}^{n}=0
$$


with boundary conditions

$$
\begin{aligned}
\lim _{S_{t}^{n} \rightarrow \infty} E_{p}^{n}\left(S_{t}^{n}, t\right) & =0 \text { on }[0, T] \\
E_{p}^{n}\left(S_{t}^{n}, T\right) & =\phi\left(S_{t}^{n}\right)=\left(K-S_{t}^{n}\right)^{+} \text {on }[0, T] \\
E_{p}^{n}(0, t) & =K e^{-r(T-t)} \text { on }[0, T]
\end{aligned}
$$

Also we have for the European power call option with non-dividend yield:

$$
\begin{gathered}
\frac{\partial E_{c}^{n}}{\partial t}+n\left(\frac{1}{2} \sigma^{2}(n-1)+r\right) S_{t}^{n} \frac{\partial E_{c}^{n}}{\partial S_{t}^{n}} \\
+\frac{1}{2}\left(\sigma n S_{t}^{n}\right)^{2} \frac{\partial^{2} E_{c}^{n}}{\partial\left(S_{t}^{n}\right)^{2}}-r E_{c}^{n}=0
\end{gathered}
$$

with boundary conditions

$$
\begin{aligned}
\lim _{S_{t}^{n} \rightarrow \infty} E_{c}^{n}\left(S_{t}^{n}, T\right) & =\infty \text { on }[0, T] \\
E_{c}^{n}\left(S_{t}^{n}, T\right) & =\phi\left(S_{t}^{n}\right)=\left(S_{t}^{n}-K\right)^{+} \text {on }[0, T] \\
E_{c}^{n}(0, t) & =0 \text { on }[0, T]
\end{aligned}
$$

The solutions to (18) and (22) can be obtained using the same procedures for solving Black-Scholes partial differential equation.

\subsection{A Review of Classic Integral Transforms}

We present here some integral transforms namely Fourier, Laplace and Mellin transforms and their inversion formulas. Under a suitable constant $c \in \Re$ and appropriate condition for the function $f$ we have the following:

- The Fourier transform and its inversion formula are given respectively by

$$
\begin{gathered}
\mathcal{F}(f(x) ; y)=\tilde{f}(y)=\int_{-\infty}^{\infty} f(x) e^{i x y} d x \\
\mathcal{F}^{-1}(\tilde{f}(y) ; x)=\tilde{f}(x)=\frac{1}{2 \pi} \int_{-\infty}^{\infty} \tilde{f}(y) e^{-i x y} d y
\end{gathered}
$$

- The Laplace transform and its inversion formulas are given respectively by

$$
\mathcal{L}(f(x) ; \theta)=\tilde{f}(\theta)=\int_{0}^{\infty} f(x) e^{-\theta x} d x
$$


and

$$
\mathcal{L}^{-1}(\tilde{f}(\theta) ; x)=f(x)=\frac{1}{2 \pi i} \int_{c-i \infty}^{c+i \infty} \tilde{f}(\theta) e^{\theta x} d \theta
$$

- The Mellin transform and its inversion formula are given respectively by

$$
\mathcal{M}(f(x) ; \omega)=\tilde{f}(\omega)=\int_{0}^{\infty} f(x) x^{\omega-1} d x
$$

and

$$
\mathcal{M}^{-1}(\tilde{f}(\omega) ; x)=f(x)=\frac{1}{2 \pi i} \int_{c-i \infty}^{c+i \infty} \tilde{f}(\omega) x^{-\omega} d \omega
$$

The Fourier transform is popular in pure mathematics and finance. The Laplace transform is a widely used integral transform in mathematics with many applications in physics and engineering. The Mellin transform is used often in statistics, in analytic number theory and in the analysis of algorithms. All the three transforms and their inversion formulas are equivalent by elementary substitution. With the change of variable $x=e^{s}$, it is observed that the Mellin transform is closely related to the Laplace transform and the Fourier transform. In particular, if $\mathcal{F}(f(x), \omega)$ and $\mathcal{L}(f(x), \omega)$ denote the two-sided Fourier and Laplace transform respectively, then we have

$$
\mathcal{M}(f(x), \omega)=\mathcal{L}\left(f\left(e^{-x}\right), \omega\right)=\mathcal{F}\left(f\left(e^{-x}\right),-i \omega\right)
$$

However, there are numerous applications where it has been established that it is more convenient to operate directly with the Mellin transform rather than the Laplace-Fourier version. Next we discuss the Mellin transform for the valuation of some vanilla options.

\section{The Mellin Transform Method for the Valuation of Some Vanilla Power Options with Non-Dividend Yield}

This section presents some operational fundamental properties and Mellin transform method for the valuation of some vanilla power options namely European and American power options with non-dividend yield.

\subsection{The Mellin Transform Method}

The Mellin transform is a complex valued function defined on a vertical strip in the $\omega$-plane whose boundaries are determined by the asymptotic behaviour 
of $f(x)$ as $x \rightarrow 0^{+}$and $x \rightarrow \infty$. Mellin transform is denoted by $\mathcal{M}(f(x), \omega)$ and defined as

$$
\mathcal{M}(f(x), \omega)=\tilde{f}(\omega)=\int_{0}^{\infty} f(x) x^{\omega-1} d x
$$

where $f(x)$ is a locally Lebesgue integrable function. The largest strip $(a, b)$ in which the integral converges is called fundamental strip. The conditions are

$$
\begin{aligned}
& f(x)=O\left(x^{u}\right), \text { for } x \rightarrow 0^{+} \\
& f(x)=O\left(x^{v}\right), \text { for } x \rightarrow \infty
\end{aligned}
$$

when $u>v$, guarantee the existence of $\mathcal{M}(f(x), \omega)$ in strip $(-u,-v)$. Thus, the existence is granted for locally integrable functions, whose exponent in the order at 0 is strongly larger than the exponent of the order at infinity. Conversely, if $f(x)$ is an integrable function with fundamental strip (a, b), then if $c$ is such that $a<c<b$ and $f(c+i t)$ is integrable, the equality in (32) holds almost everywhere on $(0, \infty)$, where $f(x)$ and $\tilde{f}(\omega)$ are known as Mellin transform pair. The inversion formula for the Mellin transform is given as

$$
\mathcal{M}^{-1}(\tilde{f}(\omega), x)=f(x)=\frac{1}{2 \pi i} \int_{c-i \infty}^{c+i \infty} \tilde{f}(\omega) x^{-\omega} d \omega
$$

Using (33) and (34) above we have the following conditions for European power put options:

$$
\begin{aligned}
& E_{p}^{n}\left(S_{t}^{n}, t\right)=O(1), \text { for } S_{t}^{n} \rightarrow 0^{+} \\
& E_{p}^{n}\left(S_{t}^{n}, t\right)=O\left(S_{t}^{n}\right), \text { for } S_{t}^{n} \rightarrow \infty
\end{aligned}
$$

The Mellin transform of $E_{p}^{n}\left(S_{t}^{n}, t\right)$ is given by

$$
\tilde{E}_{p}^{n}\left(S_{t}^{n}, t\right)=\int_{0}^{\infty} E_{p}^{n}\left(S_{t}^{n}, t\right)\left(S_{t}^{n}\right)^{\omega-1} d S_{t}^{n}
$$

where $\omega$ is a complex variable with $0<\operatorname{Re}(\omega)<\infty$. Using (35), the inversion formula of the Mellin transform is defined by

$$
E_{p}^{n}\left(S_{t}^{n}, t\right)=\frac{1}{2 \pi i} \int_{c-i \infty}^{c+i \infty} \tilde{E}_{p}^{n}\left(S_{t}^{n}, t\right)\left(S_{t}^{n}\right)^{-\omega} d \omega
$$




\subsubsection{Some Operational Fundamental Properties of the Mellin Transform}

If $\mathcal{M}(f(x), \omega)=\tilde{f}(\omega)$, then we have the following properties of the Mellin transform.

- Scaling Property

$$
\mathcal{M}(f(a x), \omega)=\int_{0}^{\infty} f(a x) x^{\omega-1} d x=a^{-\omega} \tilde{f}(\omega)
$$

e.g if $a=2$, using (40) we have

$$
\mathcal{M}(f(2 x), \omega)=\int_{0}^{\infty} f(2 x) x^{\omega-1} d x=2^{-\omega} \tilde{f}(\omega)
$$

- Shifting Property

$$
\mathcal{M}\left(x^{a} f(x), \omega\right)=\tilde{f}(\omega+a)
$$

e.g if $a=4$, using (41) we have

$$
\mathcal{M}\left(x^{4} f(x), \omega\right)=\tilde{f}(\omega+4)
$$

- Mellin Transform of Derivatives

$$
\mathcal{M}\left(\frac{d^{k}}{d x^{k}} f(x), \omega\right)=(-1)^{k}(\omega-k)_{k} \tilde{f}(\omega-k)
$$

where

$$
(\omega-k)_{k}=(\omega-k)(\omega-k+1) \ldots(\omega-1)=\frac{(\omega-1) !}{(\omega-k-1) !}=\frac{\Gamma(\omega)}{\Gamma(\omega-k)}
$$

- Derivative Multiplied by Independent Variable

$$
\mathcal{M}\left(\omega^{k} \frac{d^{k}}{d x^{k}} f(x), \omega\right)=(-1)^{k}(\omega)_{k} \tilde{f}=(-1)^{k} \frac{\Gamma(\omega+k)}{\Gamma(\omega)} \tilde{f}(\omega)_{k}
$$

e.g if $f(x)=x^{2}$, using (43) we have

$$
\mathcal{M}\left(\omega^{2} \frac{d^{2}}{d x^{2}} x^{2}, \omega\right)=\left(\omega^{2}+\omega\right) \tilde{f}(\omega)
$$


- Mellin Transform of Integrals

$$
\mathcal{M}\left(\int_{0}^{x} f(t) d t\right)=\frac{-1}{\omega} \tilde{f}(\omega+1)
$$

- Raising the independent variable to a Real Power

$$
\mathcal{M}\left(f\left(x^{a}\right), \omega\right)=\tilde{f}\left(\frac{\omega}{a}\right)
$$

- Inverse of Independent Variable

$$
\mathcal{M}\left(x^{-1} f\left(x^{-1}\right), \omega\right)=\tilde{f}(1-\omega)
$$

- Multiplication by $\ln x$

$$
\mathcal{M}((\ln x) f(x), \omega)=\frac{d}{d \omega} \tilde{f}(\omega)
$$

- Multiplication by the Power of $\ln x$

$$
\mathcal{M}\left((\ln x)^{k} f(x), \omega\right)=\frac{d^{k}}{d \omega^{k}} \tilde{f}(\omega)
$$

- Convolution Property

$$
\mathcal{M}(f(x) \cdot g(x), \omega)=\frac{1}{2 \pi i} \int_{c-i \infty}^{c+i \infty} \tilde{f}(z) \tilde{g}(\omega-z) d z
$$

- Multiplicative Convolution

$$
\begin{gathered}
\mathcal{M}(f \vee g)=\mathcal{M}\left(\int_{0}^{\infty} f\left(\frac{x}{u}\right) g(u) \frac{d u}{u} ; \omega\right)=\tilde{f}(\omega) \tilde{g}(\omega) \\
\mathcal{M}^{-1}(\tilde{f}(\omega) \tilde{g}(\omega))=\int_{0}^{\infty} f\left(\frac{x}{u}\right) g(u) \frac{d u}{u}
\end{gathered}
$$

- Parseval's Formulas

$$
\begin{gathered}
\int_{0}^{\infty} f(x) g(x) d x=\int_{c-\infty}^{c+\infty} \mathcal{M}(f ; \omega) \mathcal{M}(g ; 1-\omega) d \omega \\
\int_{0}^{\infty} f(x) g^{*}(x) x^{2 r+1} d x=\int_{c-\infty}^{c+\infty} \mathcal{M}(f)(\beta) \mathcal{M}^{*}(g)(\beta) d \beta
\end{gathered}
$$

where

$$
\mathcal{M}(f)(\beta)=\int_{0}^{\infty} f(x) x^{2 \pi \beta i+r} d x
$$

just to mention a few. 


\subsubsection{Some Integral Representations for European Power Call and Put Options Payoffs}

Consider the power call payoff with strike price $K>0$ given by (1) as

$$
E_{c}^{n}\left(S_{T}^{n}, T\right)=\max \left(S_{T}^{n}-K, 0\right)=\left(S_{T}^{n}-K\right)^{+}
$$

where $S_{T}^{n}$ is used as transform variable. An elementary calculations shows

$$
\mathcal{M}\left(E_{c}^{n}\left(S_{T}^{n}, T\right), \omega\right)=\tilde{E}_{c}^{n}(\omega, T)=\int_{0}^{\infty}\left(S_{T}^{n}-K\right)^{+}\left(S_{T}^{n}\right)^{\omega-1}=\frac{K^{\omega+1}}{\omega(\omega+1)}, \quad(\Re \omega>1)
$$

and the inversion formula gives

$$
\mathcal{M}^{-1}\left(\tilde{E}_{c}^{n}(\omega, T), S_{T}^{n}\right)=E_{c}^{n}\left(S_{t}^{n}, T\right)=\int_{c-i \infty}^{c+i \infty} \frac{K^{\omega+1}}{\omega(\omega+1)}\left(S_{t}^{n}\right)^{-\omega},\left(S_{T}^{n}>0\right)
$$

with $c>0$ an arbitrary fixed number. Similarly the power put payoff given by (2) as

$$
E_{p}^{n}\left(S_{T}^{n}, T\right)=\max \left(K-S_{T}^{n}, 0\right)=\left(K-S_{T}^{n}\right)^{+}
$$

has the same Mellin transform but the formula is valid in a different domain

$$
\mathcal{M}\left(E_{p}^{n}\left(S_{T}^{n}, T\right), \omega\right)=\tilde{E}_{p}^{n}(\omega, T)=\int_{0}^{\infty}\left(K-S_{n}^{T}\right)^{+}\left(S_{T}^{n}\right)^{\omega-1}=\frac{K^{\omega+1}}{\omega(\omega+1)},(\Re \omega>1)
$$

and the inversion formula gives

$$
\mathcal{M}^{-1}\left(\tilde{E}_{p}^{n}(\omega, T), S_{T}^{n}\right)=E_{c}^{n}\left(S_{t}^{n}, T\right)=\int_{d-i \infty}^{d+i \infty} \frac{K^{\omega+1}}{\omega(\omega+1)}\left(S_{t}^{n}\right)^{-\omega},\left(S_{T}^{n}>0\right)
$$

with $d>0$ an arbitrary fixed number. For the case $0<\Re \omega<1$, the Mellin transform and its inversion formula for power call payoff are obtained as

$$
\begin{gathered}
\mathcal{M}\left(E_{c}^{n}\left(S_{T}^{n}, T\right)-S_{T}^{n}, \omega\right)=\int_{0}^{\infty}\left(\left(S_{T}^{n}-K\right)^{+}-S_{T}^{n}\right)\left(S_{T}^{n}\right)^{\omega-1}=\frac{K^{\omega+1}}{\omega(\omega+1)}, \quad(0<\Re \omega<1) \\
E_{c}^{n}\left(S_{t}^{n}, T\right)=S_{T}^{n}+\int_{c-i \infty}^{c+i \infty} \frac{K^{\omega+1}}{\omega(\omega+1)}\left(S_{t}^{n}\right)^{-\omega},\left(S_{T}^{n}>0\right)
\end{gathered}
$$

with $0<c<1$ an arbitrary fixed number. 


\subsection{Mellin Transform Method for the Valuation of European Power Put Option with Non-Dividend Yield}

Consider the European power put option given by (18) as

$$
\frac{\partial E_{p}^{n}}{\partial t}+n\left(\frac{1}{2} \sigma^{2}(n-1)+r\right) S_{t}^{n} \frac{\partial E_{p}^{n}}{\partial S_{t}^{n}}+\frac{1}{2}\left(\sigma n S_{t}^{n}\right)^{2} \frac{\partial^{2} E_{p}^{n}}{\partial\left(S_{t}^{n}\right)^{2}}-r E_{p}^{n}=0
$$

Taking the Mellin transform of (18) we have

$$
\begin{aligned}
& \mathcal{M}\left(\frac{\partial E_{p}^{n}\left(S_{t}^{n}, t\right)}{\partial t}+n\left(\frac{1}{2} \sigma^{2}(n-1)+r\right) S_{t}^{n} \frac{\partial E_{p}^{n}\left(S_{t}^{n}, t\right)}{\partial S_{t}^{n}}\right) \\
& +\mathcal{M}\left(\frac{1}{2}\left(\sigma n S_{t}^{n}\right)^{2} \frac{\partial^{2} E_{p}^{n}\left(S_{t}^{n}, t\right)}{\partial\left(S_{t}^{n}\right)^{2}}-r E_{p}^{n}\left(S_{t}^{n}, t\right)\right)=\mathcal{M}(0)
\end{aligned}
$$

where

$$
\begin{aligned}
\mathcal{M}(0) & =0, \\
\mathcal{M}\left(E_{p}^{n}\left(S_{t}^{n}, t\right)\right. & =\tilde{E}_{p}^{n}(\omega, t), \\
\mathcal{M}\left(\frac{\partial E_{p}^{n}\left(S_{t}^{n}, t\right)}{\partial t}\right) & =\frac{\partial \tilde{E}_{p}^{n}(\omega, t)}{\partial t}, \\
\mathcal{M}\left(S_{t}^{n} \frac{\partial E_{p}^{n}\left(S_{t}^{n}, t\right)}{\partial S_{t}^{n}}\right) & =-\omega \tilde{E}_{p}^{n}(\omega, t),
\end{aligned}
$$

and

$$
\mathcal{M}\left(\frac{\partial^{2} E_{p}^{n}\left(S_{t}^{n}, t\right)}{\partial\left(S_{t}^{n}\right)^{2}}\right)=\left(\omega^{2}+\omega\right) \tilde{E}_{p}^{n}(\omega, t)
$$

Substituting the values of (61), (62), (63), (64) and (65) into (60) we have

$$
\begin{aligned}
& \frac{\partial \tilde{E}_{p}^{n}(\omega, t)}{\partial t}-n\left(\frac{1}{2} \sigma^{2}(n-1)+r\right) \omega \frac{\partial \tilde{E}_{p}^{n}(\omega, t)}{\partial \omega} \\
& +\frac{1}{2}(\sigma n)^{2}\left(\omega^{2}+\omega\right) \frac{\partial^{2} \tilde{E}_{p}^{n}(\omega, t)}{\partial \omega^{2}}-r \tilde{E}_{p}^{n}(\omega, t)=0
\end{aligned}
$$

Therefore,

$$
\frac{\partial \tilde{E}_{p}^{n}(\omega, t)}{\partial t}+\frac{n^{2} \sigma^{2}}{2}\left(\omega^{2}+\omega\left(1-\frac{n-1}{n}+\frac{2 r}{n \sigma^{2}}\right)-\frac{2 r}{n^{2} \sigma^{2}}\right) \tilde{E}_{p}^{n}(\omega, t)=0
$$


Setting $\alpha_{1}=\left(1-\frac{n-1}{n}+\frac{2 r}{n \sigma^{2}}\right)$ and $\alpha_{2}=\frac{2 r}{n^{2} \sigma^{2}}$. Then (67) yields

$$
\frac{\partial \tilde{E}_{p}^{n}(\omega, t)}{\partial t}+\frac{n^{2} \sigma^{2}}{2}\left(\omega^{2}+\omega \alpha_{1}-\alpha_{2}\right) \tilde{E}_{p}^{n}(\omega, t)=0
$$

Solving (68) integrating from 0 to $t$ using separation of variables, we have

$$
\begin{aligned}
\int_{0}^{t} \frac{\partial \tilde{E}_{p}^{n}(\omega, \tau)}{\tilde{E}_{p}^{n}(\omega, \tau)} & =\int_{0}^{t} \frac{-1}{2} n^{2} \sigma^{2}\left(\omega^{2}+\omega \alpha_{1}-\alpha_{2}\right) \partial \tau \\
\ln \left(\frac{\tilde{E}_{p}^{n}(\omega, t)}{\tilde{E}_{p}^{n}(\omega, 0)}\right) & =\frac{-1}{2} n^{2} \sigma^{2}\left(\omega^{2}+\omega \alpha_{1}-\alpha_{2}\right) t \\
\tilde{E}_{p}^{n}(\omega, t) & =\tilde{E}_{p}^{n}(\omega, 0) e^{\frac{-1}{2} n^{2} \sigma^{2}\left(\omega^{2}+\omega \alpha_{1}-\alpha_{2}\right) t}
\end{aligned}
$$

Let $\tilde{E}_{p}^{n}(\omega, 0)=c(\omega)$, where $c(\omega)$ is a constant that depends on the boundary conditions, then

$$
\tilde{E}_{p}^{n}(\omega, t)=c(\omega) e^{-\frac{1}{2} n^{2} \sigma^{2}\left(\omega^{2}+\alpha_{1} \omega-\alpha_{2}\right) t}
$$

Now let us consider the terminal condition of European power put option given by (24) which is of the form

$$
E_{p}^{n}\left(S_{t}^{n}, T\right)=\phi\left(S_{t}^{n}\right)=\left(K-S_{t}^{n}\right)^{+}=\max \left(K-S_{t}^{n}, 0\right) \text { on }[0, \infty]
$$

Then the Mellin transform of (24) is obtained as

$$
\mathcal{M}\left(E_{p}^{n}\left(S_{T}^{n}, T\right), \omega\right)=\tilde{\phi}(\omega, t)=\int_{0}^{\infty}\left(K-S_{n}^{T}\right)^{+}\left(S_{T}^{n}\right)^{\omega-1}=\frac{K^{\omega+1}}{\omega(\omega+1)}
$$

where (70) is independent of $n$. Therefore the constant $c(\omega)$ becomes

$$
c(\omega)=\tilde{\phi}(\omega, t) e^{\frac{1}{2} n^{2} \sigma^{2}\left(\omega^{2}+\alpha_{1} \omega-\alpha_{2}\right) T}
$$

Substituting (71) into (69) yields:

$$
\tilde{E}_{p}^{n}(\omega, t)=\tilde{\phi}(\omega, t) e^{-\frac{1}{2} n^{2} \sigma^{2}\left(\omega^{2}+\alpha_{1} \omega-\alpha_{2}\right)(T-t)}
$$

Using the inversion formula of Mellin transform, the price of European power put option is obtained as

$$
E_{p}^{n}\left(S_{t}^{n}, t\right)=\frac{1}{2 \pi i} \int_{c-i \omega}^{c+i \omega} \tilde{E}_{n}^{p}(\omega, t)\left(S_{t}^{n}\right)^{-\omega} d \omega
$$

Substituting (72) into (73), we have

$$
E_{p}^{n}\left(S_{t}^{n}, t\right)=\frac{1}{2 \pi i} \int_{c-i \omega}^{c+i \omega} \frac{\tilde{\phi}(\omega, t) e^{-\frac{1}{2} n^{2} \sigma^{2}\left(\omega^{2}+\alpha_{1} \omega-\alpha_{2}\right)(T-t)}}{\left(S_{t}^{n}\right)^{\omega}} d \omega
$$

where $\tilde{\phi}(\omega, t)$ is given by $(70),\left(S_{t}^{n}, t\right) \in\{(0, \infty) \times[0, T]\}, c \in(0, \infty)$ and $\{\omega \in$ $\mathbf{C} \mid 0<\operatorname{Re}(\omega)<\infty\}$ 


\subsection{Derivation of Black-Scholes Formula for the Valuation of European Power Put Option using Mellin Transform Method}

Theorem 3.3.1: Let $S_{t}^{n}$ be the price of the underlying asset, $K$ be the strike price, $r$ be the risk interest rate and $T$ be the time to maturity. Using the convolution property of the Mellin transform

$$
E_{n}^{p}\left(S_{t}^{n}, t\right)=\int_{0}^{\infty} \phi(v) \xi\left(\frac{S_{t}^{n}}{v}\right) \frac{1}{v} d v
$$

we have the Black-Scholes formula for the valuation of European power put option as

$$
\begin{gathered}
E_{n}^{p}\left(S_{t}^{n}, t\right)=K e^{-} r(T-t) N\left(d_{2, n}\left(S_{t}^{n}, K, T\right)\right) \\
-e^{\left(r(n-1)+\frac{1}{2} n(n-1) \sigma^{2}(T-t)\right)} S_{t}^{n} N\left(-d_{1, n}\left(S_{t}^{n}, K, T\right)\right)
\end{gathered}
$$

Proof: Using the convolution property of Mellin Transform method, the price of European power put option is given by

$$
E_{n}^{p}\left(S_{t}^{n}, t\right)=\int_{0}^{\infty} \phi(v) \xi\left(\frac{S_{t}^{n}}{v}\right) \frac{1}{v} d v
$$

Therefore,

$$
E_{n}^{p}\left(S_{t}^{n}, t\right)=\int_{0}^{\infty} \phi(v) \xi\left(S_{t}^{n} v^{-1}\right) v^{-1} d v
$$

where $\phi(v)$ is to be determined. Let us consider

$$
\frac{n^{2} \sigma^{2}}{2}(T-t)\left(\omega^{2}+\alpha_{1} \omega-\alpha_{2}\right)=\frac{n^{2} \sigma^{2}}{2}(T-t)\left[\left(\omega+\frac{\alpha_{1}}{2}\right)^{2}-\left(\frac{\alpha_{1}}{2}\right)^{2}-\alpha_{2}\right]
$$

Setting $\rho_{1}=\frac{n^{2} \sigma^{2}}{2}(T-t)$ on the right side of (78), therefore (78) becomes

$$
\frac{n^{2} \sigma^{2}}{2}(T-t)\left(\omega^{2}+\alpha_{1} \omega-\alpha_{2}\right)=\rho_{1}\left[\left(\omega+\frac{\alpha_{1}}{2}\right)^{2}-\left(\frac{\alpha_{1}}{2}\right)^{2}-\alpha_{2}\right]
$$

Let $\rho_{2}=\frac{\alpha_{1}}{2}$. Then (79) yields

$$
\frac{n^{2} \sigma^{2}}{2}(T-t)\left(\omega^{2}+\alpha_{1} \omega-\alpha_{2}\right)=\rho_{1}\left[\left(\omega+\rho_{2}\right)^{2}-\left(\rho_{2}\right)^{2}-\alpha_{2}\right]
$$

Thus (77) becomes

$$
E_{n}^{p}\left(S_{t}^{n}, t\right)=e^{-G} \frac{1}{2 \pi i} \int_{c-i \omega}^{c+i \omega} \tilde{\phi}(\omega, t) e^{\rho_{1}(\omega+\rho)^{2}}\left(S_{t}^{n}\right)^{-\omega} d \omega
$$


where $G=-\rho_{1}\left(\rho_{2}^{2}+\alpha_{2}\right)$. Now $\tilde{\phi}$ is the Mellin transform of

$$
e^{\rho_{1}(\omega+\rho)^{2}}=\int_{0}^{\infty} \xi\left(S_{t}^{n}\right)\left(S_{t}^{n}\right)^{\omega-1} d S_{t}^{n}
$$

Using the transformation given by

$$
e^{\phi \omega^{2}}=\frac{1}{2 \sqrt{\pi}} \int_{0}^{\infty} e^{\frac{\left(\ln S_{t}^{n}\right)^{2}}{4 \phi}}\left(S_{t}^{n}\right)^{\omega-1} d S_{t}^{n}, \quad \Re(\phi) \geq 0
$$

we get

$$
\xi\left(S_{t}^{n}\right)=\xi\left(S_{t}^{n}, t\right)=\frac{\left(S_{t}^{n}\right)^{\rho_{2}}}{n \sigma \sqrt{2 \pi(T-t)}} e^{\frac{-1}{2}\left(\frac{\ln S_{t}^{n}}{n \sigma \sqrt{T-t}}\right)^{2}}
$$

The European power put option can therefore be expressed as

$$
\begin{gathered}
E_{n}^{p}\left(S_{t}^{n}, t\right)=e^{\frac{-G}{n \sigma \sqrt{2 \pi(T-t)}}} \int_{0}^{K}(K-v)\left(\frac{S_{t}^{n}}{v}\right)^{\rho_{2}} \exp \left(\left.\frac{-1}{2}\left(\frac{\ln \left(\frac{S_{t}^{n}}{v}\right)}{n \sigma \sqrt{T-t}}\right)\right|^{2} d v\right. \\
E_{n}^{p}\left(S_{t}^{n}, t\right)=e^{\frac{-G}{n \sigma \sqrt{2 \pi(T-t)}}} \int_{0}^{K} K\left(\frac{S_{t}^{n}}{v}\right)^{\rho_{2}} \exp \left(\frac{-1}{2}\left(\frac{\ln \left(\frac{S_{t}^{n}}{v}\right)}{n \sigma \sqrt{T-t}}\right)\right)^{2} d v \\
-e^{\frac{-G}{n \sigma \sqrt{2 \pi(T-t)}}} \int_{0}^{K} v\left(\frac{S_{t}^{n}}{v}\right)^{\rho_{2}} \exp \left(\frac{-1}{2}\left(\frac{\ln \left(\frac{S_{t}^{n}}{v}\right)}{n \sigma \sqrt{T-t}}\right) \mid \frac{1}{v} d v\right.
\end{gathered}
$$

Let $\hat{\Omega}_{1}=e^{\frac{-G}{n \sigma \sqrt{2 \pi(T-t)}}}\left(\Omega_{1}\right)$ and $\hat{\Omega}_{2}=-e^{\frac{-G}{n \sigma \sqrt{2 \pi(T-t)}}}\left(\Omega_{2}\right)$ denote the first and second terms of (86), then we write (86) as

$$
E_{n}^{p}\left(S_{t}^{n}, t\right)=\hat{\Omega}_{1}+\hat{\Omega}_{2}=e^{\frac{-G}{n \sigma \sqrt{2 \pi(T-t)}}}\left(\Omega_{1}-\Omega_{2}\right)
$$

where

$$
\Omega_{1}=\int_{0}^{K} K\left(\frac{S_{t}^{n}}{v}\right)^{\rho_{2}} \exp \left(\frac{-1}{2}\left(\frac{\ln \left(\frac{S_{t}^{n}}{v}\right)}{n \sigma \sqrt{T-t}}\right)\right)^{2} d v
$$

and

$$
\Omega_{2}=\int_{0}^{K} v\left(\frac{S_{t}^{n}}{v}\right)^{\rho_{2}} \exp \left(\frac{-1}{2}\left(\frac{\ln \left(\frac{S_{t}^{n}}{v}\right)}{n \sigma \sqrt{T-t}}\right)\right)^{2} d v
$$


denote the first and second integrals in (86) respectively. We use the following transformations to evaluate the first and second integrals in (86) respectively:

$$
\begin{aligned}
\lambda & =\frac{1}{n \sigma \sqrt{T-t}}\left(\ln \left(\frac{S_{t}^{n}}{v}\right)-\left(\rho_{2} n^{2} \sigma^{2}\right)(T-t)\right) \\
\tilde{\lambda} & =\frac{1}{n \sigma \sqrt{T-t}}\left(\ln \left(\frac{S_{t}^{n}}{v}\right)-\left(\rho_{2}-1\right) n^{2} \sigma^{2}(T-t)\right)
\end{aligned}
$$

Solving further, the first term of (86) is obtained as

$$
\hat{\Omega}_{1}=K e^{-} r(T-t) N\left(d_{2, n}\left(S_{t}^{n}, K, T\right)\right)
$$

where

$$
d_{2, n}\left(S_{t}^{n}, K, T\right)=\frac{\ln \left(\frac{S_{t}^{n}}{K}\right)+n\left(r-\frac{\sigma^{2}}{2}\right)(T-t)}{n \sigma \sqrt{T-t}}
$$

Similarly, the second term is evaluated as

$$
\hat{\Omega}_{2}=-e^{\left(r(n-1)+\frac{1}{2} n(n-1) \sigma^{2}(T-t)\right)} S_{t}^{n} N\left(-d_{1, n}\left(S_{t}^{n}, K, T\right)\right)
$$

where

$$
d_{1, n}\left(S_{t}^{n}, K, T\right)=\frac{\ln \left(\frac{S_{t}^{n}}{K}\right)+n\left(r+\left(n-\frac{\sigma^{2}}{2}\right)\right)(T-t)}{n \sigma \sqrt{T-t}}
$$

We denote the distribution function for a normal variable by $N(x)$

$$
N(x)=\frac{1}{\sqrt{2 \pi}} \int_{-\infty}^{x} e^{-\frac{u^{2}}{2}} d u
$$

Substituting (92) and (94) into (87), we have

$$
\begin{gathered}
E_{n}^{p}\left(S_{t}^{n}, t\right)=K e^{-} r(T-t) N\left(d_{2, n}\left(S_{t}^{n}, K, T\right)\right) \\
-e^{\left(r(n-1)+\frac{1}{2} n(n-1) \sigma^{2}(T-t)\right)} S_{t}^{n} N\left(-d_{1, n}\left(S_{t}^{n}, K, T\right)\right)
\end{gathered}
$$

(97) is called the analytical pricing formula for European power put option with non-dividend yield is given by with

$$
d_{1, n}\left(S_{t}^{n}, K, T\right)=d_{1, n}
$$

and

$$
d_{2, n}\left(S_{t}^{n}, K, T\right)=d_{2, n}=d_{1, n}-n \sigma \sqrt{T-t}
$$

as given above.

\section{Remark 3.3.1:}


- This result shows the Black-Scholes formula for the European power put option is obtained using the convolution property of the Mellin transform.

- For $n=1,(97)$ becomes analytical pricing formula "the so called BlackScholes model" for European put option given by

$$
E_{1}^{p}\left(S_{t}^{1}, t\right)=K e^{-} r(T-t) N\left(d_{2,1}\left(S_{t}^{1}, K, T\right)\right)-S_{t}^{n} N\left(-d_{1,1}\left(S_{t}^{1}, K, T\right)\right)
$$

with

$$
d_{1,1}\left(S_{t}^{1}, K, T\right)=\frac{\ln \left(\frac{S_{t}^{1}}{K}\right)+\left(r+\frac{\sigma^{2}}{2}\right)(T-t)}{\sigma \sqrt{T-t}}
$$

and

$$
d_{2,1}\left(S_{t}^{1}, K, T\right)=\frac{\ln \left(\frac{S_{t}^{1}}{K}\right)+\left(r-\frac{\sigma^{2}}{2}\right)(T-t)}{\sigma \sqrt{T-t}}
$$

\subsection{The Mellin Transform Method for the Valuation of American Power Put Option with Non-Dividend Yield}

The key to determining the value of the American power option is to find the critical underlying price of the asset, which specifies the conditions under which the option should be exercised prior to maturity. The difference between European and American power options is that an American power option can be exercised by its holder at any time on or before the expiry date. This early exercise feature makes the pricing of American power option mathematically challenging and created great field of research throughout the last three decades. The valuation of American power option can be seen under several mathematical aspects, leading to different but equivalent mathematical formulations of the problem. In this paper we will make use of free boundary formulation for the valuation of American power put option with non-dividend yield.

We extend the domain of the Black-Scholes partial differential equation by setting the final time condition $A_{p}^{n}\left(S_{t}^{n}, t\right)=A_{p}^{n}=\left(K-S_{t}^{n}\right)^{+}$for $S_{t}^{n}<\hat{S}_{t}^{n}$, where the free boundary $\hat{S}_{t}^{n}$ depends on the expiry time $T$. The non-homogeneous Black-Scholes equation for the price of American power put option with nondividend yield is given by

$$
\begin{aligned}
& \frac{\partial A_{p}^{n}\left(S_{t}^{n}, T\right)}{\partial t}+n\left(\frac{1}{2} \sigma^{2}(n-1)+r\right) S_{t}^{n} \frac{\partial A_{p}^{n}\left(S_{t}^{n}, T\right)}{\partial S_{t}^{n}} \\
& +\frac{1}{2}\left(\sigma n S_{t}^{n}\right)^{2} \frac{\partial^{2} A_{p}^{n}\left(S_{t}^{n}, T\right)}{\partial\left(S_{t}^{n}\right)^{2}}-r A_{p}^{n}\left(S_{t}^{n}, T\right)=f\left(S_{t}^{n}, t\right)
\end{aligned}
$$


with

$$
\begin{gathered}
f\left(S_{t}^{n}\right)=-r K, \quad \text { if } 0<S_{t}^{n} \leq \hat{S}_{t}^{n} \\
f\left(S_{t}^{n}\right)=0, \quad \text { if } S_{t}^{n}>\hat{S}_{t}^{n}
\end{gathered}
$$

on $(0, \infty) \times[0, T)$. The boundary conditions are given by

$$
\begin{aligned}
\lim _{S_{t}^{n} \rightarrow \infty} A_{p}^{n}\left(S_{t}^{n}, t\right) & =0 \text { on }[0, T] \\
A_{p}^{n}\left(S_{t}^{n}, T\right) & =\phi\left(S_{t}^{n}\right)=\left(K-S_{T}^{n}\right)^{+} \text {on }[0, T] \\
A_{p}^{n}(0, t) & =K \text { on }[0, T]
\end{aligned}
$$

According to Wilmott et al [18], arbitrage arguments show that the option's price must satisfy smooth pasting conditions at the free boundary $\hat{S}_{t}^{n}$. We have the payoff for the American power put option as

$$
A_{p}^{n}\left(\hat{S}_{t}^{n}, t\right)=K-\hat{S}_{t}^{n}
$$

Differentiating (109) with respect to $S_{t}^{n}=\hat{S}_{t}^{n}$, we have

$$
\frac{\partial A_{p}^{n}\left(\hat{S}_{t}^{n}, t\right)}{\partial \hat{S}_{t}^{n}}=-1
$$

The Mellin transform of the American power put option in (103) is obtained as

$$
\frac{\partial \tilde{A}_{p}^{n}(\omega, t)}{\partial t}+\frac{n^{2} \sigma^{2}}{2}\left(\omega^{2}+\omega\left(1-\frac{n-1}{n}+\frac{2 r}{n \sigma^{2}}\right)-\frac{2 r}{n^{2} \sigma^{2}}\right) \tilde{A}_{p}^{n}(\omega, t)=\tilde{f}(\omega, t)
$$

Setting $\alpha_{1}=\left(1-\frac{n-1}{n}+\frac{2 r}{n \sigma^{2}}\right)$ and $\alpha_{2}=\frac{2 r}{n^{2} \sigma^{2}}$. Then (110) yields

$$
\frac{\partial \tilde{A}_{p}^{n}(\omega, t)}{\partial t}+\frac{n^{2} \sigma^{2}}{2}\left(\omega^{2}+\omega \alpha_{1}-\alpha_{2}\right) \tilde{A}_{p}^{n}(\omega, t)=\tilde{f}(\omega, t)
$$

From the definition of Mellin transform, the hand side of (111) is given by

$$
\tilde{f}(\omega, t)=\int_{0}^{\infty} f\left(S_{t}^{n}, t\right)\left(S_{t}^{n}\right)^{\omega-1} d S_{t}^{n}=\frac{-r K\left(\hat{S}_{t}^{n}\right)^{\omega}}{\omega}
$$

Solving further, we have the particular solution of (112) as

$$
P_{\text {sol }}=\int_{t}^{T} \frac{-r K\left(\hat{S}_{t}^{n}\right)^{\omega}}{\omega} e^{\frac{1}{2} n^{2} \sigma^{2}\left(\omega^{2}+\alpha_{1} \omega-\alpha_{2}\right)(y-t)} d y
$$


The solution to the right hand side of (112) is called the complementary solution which is obtained as

$$
\tilde{A}_{p}^{n}(\omega, t)_{\text {comp.sol }}=c(\omega) e^{-\frac{1}{2} n^{2} \sigma^{2}\left(\omega^{2}+\alpha_{1} \omega-\alpha_{2}\right) t}
$$

where

$$
c(\omega)=\tilde{\phi}(\omega, t) e^{\frac{1}{2} n^{2} \sigma^{2}\left(\omega^{2}+\alpha_{1} \omega-\alpha_{2}\right) T}
$$

and

$$
\tilde{\phi}(\omega, t)=\int_{0}^{\infty}\left(K-S_{n}^{T}\right)^{+}\left(S_{T}^{n}\right)^{\omega-1}=\frac{K^{\omega+1}}{\omega(\omega+1)}
$$

The equation (114) above is the same as the Mellin transform of European power put price given by (69). Hence the general solution to (112) is given by

$$
\begin{gathered}
\tilde{A}_{p}^{n}(\omega, t)=\tilde{A}_{p}^{n}(\omega, t)_{\text {comp.sol }}+P_{\text {sol }}=\frac{K^{\omega+1}}{\omega(\omega+1)} e^{-\frac{1}{2} n^{2} \sigma^{2}\left(\omega^{2}+\alpha_{1} \omega-\alpha_{2}\right)(T-t)} \\
-\int_{t}^{T} \frac{r K\left(\hat{S}_{t}^{n}\right)^{\omega}}{\omega} e^{\frac{1}{2} n^{2} \sigma^{2}\left(\omega^{2}+\alpha_{1} \omega-\alpha_{2}\right)(y-t)} d y
\end{gathered}
$$

Using Mellin inversion formula, (115) becomes

$$
\begin{aligned}
& A_{p}^{n}(\omega, t)=\frac{1}{2 \pi i} \int_{c-i \omega}^{c+i \omega} \frac{K^{\omega+1}}{\omega(\omega+1)} e^{-\frac{1}{2} n^{2} \sigma^{2}\left(\omega^{2}+\alpha_{1} \omega-\alpha_{2}\right)(T-t)}\left(S_{t}^{n}\right)^{\omega} d \omega \\
& +\frac{r K}{2 \pi i} \int_{c-i \omega}^{c+i \omega}\left(S_{t}^{n}\right)^{-\omega} \int_{t}^{T} \frac{\left(\hat{S}_{t}^{n}(y)\right)^{\omega}}{\omega} e^{\frac{1}{2} n^{2} \sigma^{2}\left(\omega^{2}+\alpha_{1} \omega-\alpha_{2}\right)(y-t)} d y d \omega
\end{aligned}
$$

where $\left(S_{t}^{n}, t\right) \in\{(0, \infty) \times[0, T]\}, c \in(0, \infty)$ and $\{\omega \in \mathbf{C} \mid 0<R e(\omega)<\infty\}$. Let $S_{t}^{n}=\hat{S}_{t}^{n}$ in (116) and using (109) and (110), we have the integral equation for free boundary formulation

$K-\hat{S}_{t}^{n}=A_{p}^{n}\left(\hat{S}_{t}^{n}, t\right)+\frac{r K}{2 \pi i} \int_{c-i \omega}^{c+i \omega}\left(\hat{S}_{t}^{n}\right)^{-\omega} \int_{t}^{T} \frac{\left(\hat{S}_{t}^{n}(y)\right)^{\omega}}{\omega} e^{\frac{1}{2} n^{2} \sigma^{2}\left(\omega^{2}+\alpha_{1} \omega-\alpha_{2}\right)(y-t)} d y d \omega$

Remark 3.4.1: For $n=1$, (115) and (116) become the Mellin transform and its inversion formula for the price of regular American put option with nondividend yield respectively. The following theorem presents a simple analytical approximation for the critical underlying price of the asset $\hat{S}_{t}^{n}$ of American power option. 
Theorem 3.4.1 [7]: If the underlying price of the asset $S_{t}^{n}$ pays no dividends, the critical underlying price of the asset $\hat{S}_{t}^{n}$ of an American power put option can be determined approximately by solving the implicit equation below

$$
\hat{S}_{t}^{n}=\frac{(2 N(\sqrt{\delta t})-1) S_{t}^{n} \alpha_{2}}{N\left(d_{1, n}\left(\hat{S}_{t}^{n}, K, T\right)\right)\left(\alpha_{2}+1\right)}
$$

where $\delta=\left(\frac{1}{2} \sigma\left(1+\alpha_{2}\right)\right)^{2}, \alpha_{2}=\frac{2 r}{n^{2} \sigma^{2}}$ and $d_{1, n}\left(\hat{S}_{t}^{n}, K, T\right)$ is given by (95).

\section{Numerical Experiment}

This section presents numerical experiment and discussion of results for the valuation of American power put option for $n=1$ with non-dividend yield using Mellin transform method. For the European power put option the Mellin transform approach is simple and straight forward.

\subsection{Example}

We consider the valuation of American power put option for $n=1$ which pays no dividend yield with the following parameters:

$S=40, K=\{35,40,45\}, T=\{0.0833,0.3333,0.5833\}, r=0.0488, \sigma=\{0.2,0.4\}$

The values of the Mellin transform columns in the Tables below are obtained from [15]. The results generated are shown in the Tables 1, 2, 3, 4, 5 and 6.

\subsection{Discussion of Results}

We compare the performance of the Mellin transform method for the valuation of American put option which pays no dividend yield with other numerical methods namely binomial model [3] with 500 time steps and Crank Nicolson finite difference method [2] with $S_{\max }=100,500$ spatial and time grids against Black-Scholes model. Tables 1, 2, 3, 4, 5 and 6 show the variation of the price of the American put option via Mellin transform method, binomial model, Crank Nicolson finite difference method and Black-Scholes analytical pricing formula with the volatility $\sigma=\{0.2,0.4\}$, maturity time $T=\{0.0833,0.3333,0.5833\}$ and exercise price $K=\{35,40,45\}$ and the fixed parameters $S=40, r=0.0488$ and $c=2$. The results generated in the Tables below demonstrate that the Mellin transform method is mutually consistent, perform very well, accurate 
and agree with the values of Black-Scholes model (BSM) [1], Crank Nicolson finite difference method [2] and binomial model. It can be seen from the Tables below that the higher the volatility, the higher the price of American put option. Also the longer the time to expiration, the longer the time the asset price has to move until it is in the money. Hence the Mellin transform method [15] is accurate, easy to implement and is a better alternative technique to other approaches like the Crank Nicolson finite difference method [2] and binomial model [3] for the valuation of American put option on a non-dividend yield.

\section{Conclusion}

In this paper, we have considered the Mellin transform method for the valuation of some vanilla power options. We have established a formula for the valuation of European power put option using the Mellin transform method on a non-dividend yield consisting of single integral. We also generalize the results of Panini R. and Srivastav R.P. [14] to derive the valuation formula for the price and free boundary of American power put option with non-dividend yield. We also show how to derive the Black-Scholes valuation formula for European power put option. To provide a sufficient numerical analysis, we have compared the results generated by the Mellin transform method for the valuation of American power put option for $n=1$ to two other numerical methods the Crank Nicolson finite difference method [2] and binomial model [3] in the context of Black-Scholes model [1]. The numerical experiment has shown that the Mellin transform method is accurate, flexible, efficient and produces accurate prices for the critical stock price for a wide range of parameter combinations. The Mellin transform method is also a powerful technique for the valuation of more complex vanilla options and some path dependent options and can be extended to jump diffusion processes, stochastic volatility and other stochastic processes but modification is required on the Mellin transform method for the valuation of call options [8].

\section{References}

[1] Black F. and Scholes M. (1973), The Pricing of Options and Corporate Liabilities. Journal of Political Economy, Vol. 81, No. 3, 637-654.

[2] Brennan M. and Schwartz E. (1978), Finite Difference Methods and Jump Processes Arising in the Pricing of Contingent Claims, Journal of Financial and Quantitative Analysis, Vol. 5(4), 461-474. 
[3] Cox J., Ross S. and Rubinstein M. (1979), Option Pricing: A Simplified Approach, Journal of Financial Economics, Vol. 7, 229-263.

[4] Fadugba S.E., Ajayi A.O. and Nwozo C.R. (2013), Effect of Volatility on Binomial Model for the Valuation of American Options, International Journal of Pure and Applied Sciences and Technology, Vol. 18, No. 1, 43-53.

[5] Fadugba S.E. and C.R. Nwozo (2014): On the Comparative Study of Some Numerical Methods for Vanilla Option Valuation, Communication in Applied Sciences, Vol. 2, No. 1.

[6] Flajolet P., Gourdon X. and Dumas P. (1995), Mellin Transforms and Asymptotics Harmonic Sums, Theoretical Computer Science, Vol. 144, 358.

[7] Frontczak R. and Schöbel R. (2008), Pricing American Options with Mellin Transforms, Working Paper.

[8] Frontczak R. and Schöbel R. (2009), On Modified Mellin Transforms, Gauss-Laguerre Quadrature and the Valuation of American Call Options, Tübinger Diskussionsbeitrag, No. 320.

[9] Gradshteyn I. and Ryshik I, (2007), Table of Integrals Series and Products, Seventh Editon, academic Press.

[10] Nwozo C.R. and Fadugba S.E. (2014), Performance Measure of Laplace Transforms for Pricing Path Dependent Options, International Journal of Pure and Applied Mathematics, Vol. 94, No. 2, 175-197, dx.doi.org/10.12732/ijpam.v94i2.5.

[11] Nwozo C.R. and Fadugba S.E. (2012), Some Numerical Methods for Options Valuation, JAMB, Communications in Mathematical Finance, Scienpress Ltd, Vol. 1, No. 1, 51-74.

[12] Nwozo C.R. and Fadugba S.E. (2012), Monte Carlo Method for Pricing Some Path Dependent Options, International Journal of Applied Mathematics, Vol. 25, No. 6, 763-778.

[13] Nwozo C.R. and Fadugba S.E. (2014), On the Accuracy of Binomial Model for the Valuation of Standard Options with Dividend Yield in the Context of Black-Scholes Model, IAENG International Journal of Applied Mathematics, Vol. 44, No. 1, 33-44. 
[14] Panini R. and Srivastav R.P. (2004), Pricing Perpectual Options using Mellin Transforms, Applied Mathematics Letters, Vol. 18, 471-474, doi: 10.1016/j.aml.2004.03.012.

[15] Panini R. and Srivastav R.P. (2004), Option Pricing with Mellin Transforms, Mathematical and Computer Modelling, Vol. 40, 43-56, doi:10.1016/j.mcm.2004.07.008.

[16] Samuelson P.E. (1965), Rational Theory of Warrant Pricing,Industrial Management Review, Vol. 6, 13-31.

[17] Vasilieva O. (2009), A New Method of Pricing Multi-Options using Mellin Transforms and Integral Equations, Master's Thesis in Financial Mathematics, School of Information Science, Computer and Electrical Engineering, Halmsta University.

[18] Wilmott P., Dewynne J. and Howison S. (1993), Option Pricng, Mathematical Models and Computation, Oxford Financial Press.

[19] Yakubovich S.B. and Nguyen T.H. (1991), The Double Mellin-Barnes Type Integrals and their Applications to Convolution Theory, Series on Soviet Mathematics, World Scientific, 199.

[20] Zemanian, A.H., (1987), Generalized Integral Transformations, Dover Publications, New York.

[21] Zieneb A.E. and Rokiah R.A. (2011), Analytical Solution for an Arithmetic Asian Option using Mellin Transforms, Vol. 5, 1259-1265. 


\section{Appendix: Table of Results}

The comparative results analysis are shown in the Tables below:

Table 1: The Price of American Put Option using $\sigma=0.2, r=$ $0.0488, S=40$

\begin{tabular}{llllll}
\hline K & T & BSM & Mellin T. & Binomial M. & Crank N. Method \\
\hline 35 & 0.0833 & 0.0062 & 0.0065 & 0.0062 & 0.0062 \\
40 & 0.0833 & 0.8403 & 0.8516 & 0.8526 & 0.8394 \\
45 & 0.0833 & 4.8399 & 5.0305 & 5.0000 & 4.8400 \\
\hline
\end{tabular}

Table 2: The Price of American Put Option using $\sigma=0.2, r=$ $0.0488, S=40$

\begin{tabular}{llllll}
\hline K & T & BSM & Mellin T. & Binomial M. & Crank N. Method \\
\hline 35 & 0.3333 & 0.1960 & 0.2014 & 0.2002 & 0.1958 \\
40 & 0.3333 & 1.5221 & 1.5792 & 1.5799 & 1.5216 \\
45 & 0.3333 & 4.7804 & 5.0846 & 5.0886 & 4.7802 \\
\hline \multicolumn{7}{c}{ Table 3. }
\end{tabular}

Table 3: The Price of American Put Option using $\sigma=0.2, r=$ $0.0488, S=40$

\begin{tabular}{llllll}
\hline K & T & BSM & Mellin T. & Binomial M. & Crank N. Method \\
\hline 35 & 0.5833 & 0.4170 & 0.4346 & 0.4330 & 0.4168 \\
40 & 0.5833 & 1.8812 & 1.9904 & 1.9908 & 1.8809 \\
45 & 0.5833 & 4.8400 & 5.2638 & 5.2670 & 4.8399 \\
\hline
\end{tabular}

Table 4: The Price of American Put Option using $\sigma=0.4, r=$ $0.0488, S=40$

\begin{tabular}{llllll}
\hline K & T & BSM. & Mellin T. & Binomial M. & Crank N. Method \\
\hline 35 & 0.0833 & 0.2456 & 0.2468 & 0.2467 & 0.2455 \\
40 & 0.0833 & 1.7575 & 1.7681 & 1.7689 & 1.7571 \\
45 & 0.0833 & 5.2360 & 5.2860 & 5.2871 & 5.2358 \\
\hline
\end{tabular}

Table 5: The Price of American Put Option using $\sigma=0.4, r=$ $0.0488, S=40$ 


\begin{tabular}{llllll}
\hline K & T & BSM & Mellin T. & Binomial M. & Crank N. Method \\
\hline 35 & 0.3333 & 1.3287 & 1.3470 & 1.3458 & 1.3295 \\
40 & 0.3333 & 3.3336 & 3.3879 & 3.3889 & 3.3334 \\
45 & 0.3333 & 6.3767 & 6.5095 & 6.5112 & 6.3765 \\
\hline
\end{tabular}

Table 6: The Price of American Put Option using $\sigma=0.4, r=$ 0.0488, $S=40$

\begin{tabular}{llllll}
\hline K & T & BSM & Mellin T. & Binomial M. & Crank N. Method \\
\hline 35 & 0.5833 & 2.1127 & 2.1568 & 2.1555 & 2.1126 \\
40 & 0.5833 & 4.2473 & 4.3543 & 4.3545 & 4.2472 \\
45 & 0.5833 & 7.1654 & 7.3840 & 7.3830 & 7.1653 \\
\hline
\end{tabular}

